\title{
Scedosporium spp.: Chronicle of an emerging pathogen
}

\author{
Carolina E. Luna-Rodríguez, Rogelio de J. Treviño-Rangel, Alexandra M. Montoya, \\ Miguel A. Becerril-García, Ángel Andrade and Gloria M. González* \\ Department of Microbiology, School of Medicine, Autonomous University of Nuevo Leon, Monterrey, Nuevo Leon, Mexico
}

\begin{abstract}
Scedosporium spp. are environmental fungi that have acquired clinical importance over the last decades. Currently, these fungi are considered emerging pathogens that affect both immunocompromised and immunocompetent people. The most serious conditions within scedosporiosis are infections with dissemination to the central nervous system, which have a mortality rate of up to $75 \%$. These fungi present intrinsic resistance to several antifungals, making options in the treatment of scedosporiosis difficult. The frequency of these infections is thought to be underestimated since the signs and symptoms are nonspecific. This leads to the suspicion of other fungal etiological agents that occur more frequently in human pathologies, such as Aspergillus spp. Currently, these five species are recognized as causing important diseases in humans: Scedosporium apiospermum, Scedosporium boydii, Scedosporium aurantiacum, Scedosporium minutisporum, and Scedosporium dehoogii.
\end{abstract}

Key words: Scedosporiosis. Emerging pathogen. Clinical mycology. Antifungal resistance.

\section{Introduction}

Over the past decades, reports of new cases of infections caused by species of the genus Scedosporium have increased at an alarming rate worldwide. The group of diseases caused by this fungus is known as scedosporiosis and may affect different parts of the human body, producing a broad spectrum of clinical manifestations ranging from a superficial localized infection to a disseminated invasive infection. The populations at greater risk are those with cystic fibrosis, organ transplant recipients, and individuals with HIV/ AIDS. Localized superficial infections generally occur in immunocompetent individuals.

Environmental factors such as natural disasters have increased the number of reports of disseminated invasive infections after the aspiration of contaminated waters in healthy individuals.
For the classification of fungi, the traditional methods involve the characteristics of the fungal colony, some physiological tests (thermotolerance, growth in cycloheximide, etc.), and microscopic characteristics of the fungal structure (especially, conidia or spores) developed in standard cultures used in mycology laboratories. Under these criteria, two species of the Scedosporium genus were first described - Scedosporium apiospermum and Scedosporium inflatum (as an anamorphous or asexual state) and Pseudallescheria boydii (as a teleomorph or sexual state) 1 .

The taxonomy of this genus has presented major changes since its first description in Italy in 1911. Moreover, a significant number of new species have been described recently, as well as a reclassification of others (Table 1). Currently, five species are recognized as the main agents responsible for scedosporiosis: CC BY-NC-ND license (http://creativecommons.org/licenses/by-nc-nd/4.0/).
} 
Table 1. Changes in the taxonomy of the genus Scedosporium throughout history

\begin{tabular}{|c|c|c|}
\hline Year & Investigator & Descriptions and proposals \\
\hline 1911 & Saccardo PA & $\begin{array}{l}\text { Described a new fungus from a sample of mycetoma: Monosporium } \\
\text { apiospermum (anamorphic phase) } \\
\text { Suggested the name Scedosporium for the same fungus }\end{array}$ \\
\hline 1919 & Castellani A and Chalmers AJ & Validated the name Scedosporium \\
\hline 1922 & Shear CL & Described a new ascomycete Allescheria boydi (anamorphic and teleomorphic phase) \\
\hline 1944 & Emmons CW & Showed that Monosporium apiospermum was an anamorph of Allescheria boydii \\
\hline \multirow[t]{2}{*}{1970} & Malloch D & $\begin{array}{l}\text { Reclassified the teleomorphic phase as Petriellidium boydii recognized Petriellidium as } \\
\text { synonymous with Pseudallescheria }\end{array}$ \\
\hline & Hughes & Recognized Scedosporium apiospermum as an anamorph of Pseudallescheria boydii \\
\hline 1984 & Malloch D and Salkin IA & Described a new species of Scedosporium: Scedosporium inflatum \\
\hline 1991 & Gueho E and De Hoog GS & Proposed that Scedosporium inflatum and Lomentospora prolificans were synonyms \\
\hline 1994 & Lennon PA & $\begin{array}{l}\text { Proposed that the name Scedosporium prolificans should include isolates of } \\
\text { Scedosporium inflatum and Lomentospora prolificans }\end{array}$ \\
\hline 2005 & $\begin{array}{l}\text { Gilgado F, Cano J, Gené J and } \\
\text { Guarro J }\end{array}$ & $\begin{array}{l}\text { Described new species: Pseudallescheria minutispora and Scedosporium aurantiacum } \\
\text { Described the complex Pseudallescheria boydii: Pseudallescheria angusta, } \\
\text { Pseudallescheria ellipsoidea, and Pseudallescheria fusoidea }\end{array}$ \\
\hline 2006 & Rainer $\mathrm{J}$ and De Hoog $\mathrm{S}$ & $\begin{array}{l}\text { Demonstrated that Scedosporium prolificans is a different species from the } \\
\text { Pseudallescheria boydii complex }\end{array}$ \\
\hline 2008 & $\begin{array}{l}\text { Gilgado F, Cano J, Gené J, } \\
\text { Sutton DA and Guarro J }\end{array}$ & $\begin{array}{l}\text { Described a new species: Scedosporium dehoogii. Showed that Scedosporium } \\
\text { apiospermum is different from Pseudallescheria boydii } \\
\text { Proposed the name of Scedosporium boydii for the anamorph of Pseudallescheria boydii } \\
\text { Modified the name of the Pseudallescheria boydii complex by Pseudallescheria/ } \\
\text { Scedosporium complex }\end{array}$ \\
\hline \multirow[t]{2}{*}{2010} & $\begin{array}{l}\text { Gilgado F, Cano J, Gené J and } \\
\text { Guarro J }\end{array}$ & $\begin{array}{l}\text { Described the teleomorphic phase of Scedosporium apiospermum: Pseudallescheria } \\
\text { apiosperma }\end{array}$ \\
\hline & Rainer and Kaltseis & Described a new species: Scedosporium deficiens \\
\hline 2014 & $\begin{array}{l}\text { Members of the ECMM/ } \\
\text { ISHAM }\end{array}$ & $\begin{array}{l}\text { Reclassified Scedosporium prolificans as Lomentospora prolificans. Proposed the } \\
\text { Scedosporium apiospermum complex: Scedosporium apiospermum, Scedosporium } \\
\text { aurantiacum, Scedosporium dehoogii, Scedosporium boydii, Scedosporium } \\
\text { minutisporum, and Scedosporium desertorum }\end{array}$ \\
\hline 2015 & ISHAM & $\begin{array}{l}\text { Proposed rearrangement of the Scedosporium apiospermum complex: Scedosporium } \\
\text { apiospermum, Scedosporium aurantiacum, Scedosporium dehoogii, Scedosporium } \\
\text { boydii, Scedosporium minutisporum, Scedosporium ellipsoideum, and Scedosporium } \\
\text { angustum }\end{array}$ \\
\hline 2016 & Chen et al. & $\begin{array}{l}\text { Proposed Scedosporium apiospermum, Scedosporium boydii, and Pseudallescheria } \\
\text { angusta as members of the Scedosporium apiospermum complex }\end{array}$ \\
\hline 2016 & $\begin{array}{l}\text { Rougeron, Stchigel, S. Giraud, } \\
\text { Bouchara and Cano }\end{array}$ & Described a new species: Scedosporium cereisporum \\
\hline 2018 & Ramirez-Garcia, et al. & $\begin{array}{l}\text { Proposed as members of the Scedosporium apiospermum complex: Scedosporium } \\
\text { angustum, Scedosporium apiospermum, Scedosporium boydii, Scedosporium } \\
\text { ellipsoideum, and Scedosporium fusarium }\end{array}$ \\
\hline
\end{tabular}

ISHAM: International Society of Human and Animal Mycology, ECMM: European Confederation of Medical Mycology.

\section{S. apiospermum, Scedosporium boydii, Scedosporium aurantiacum, Scedosporium dehoogii, and Scedospo- rium minutisporum.}

Species of the Scedosporium genus have macroscopic and microscopic characteristics which are very similar among them. These fungi have the ability to grow under 


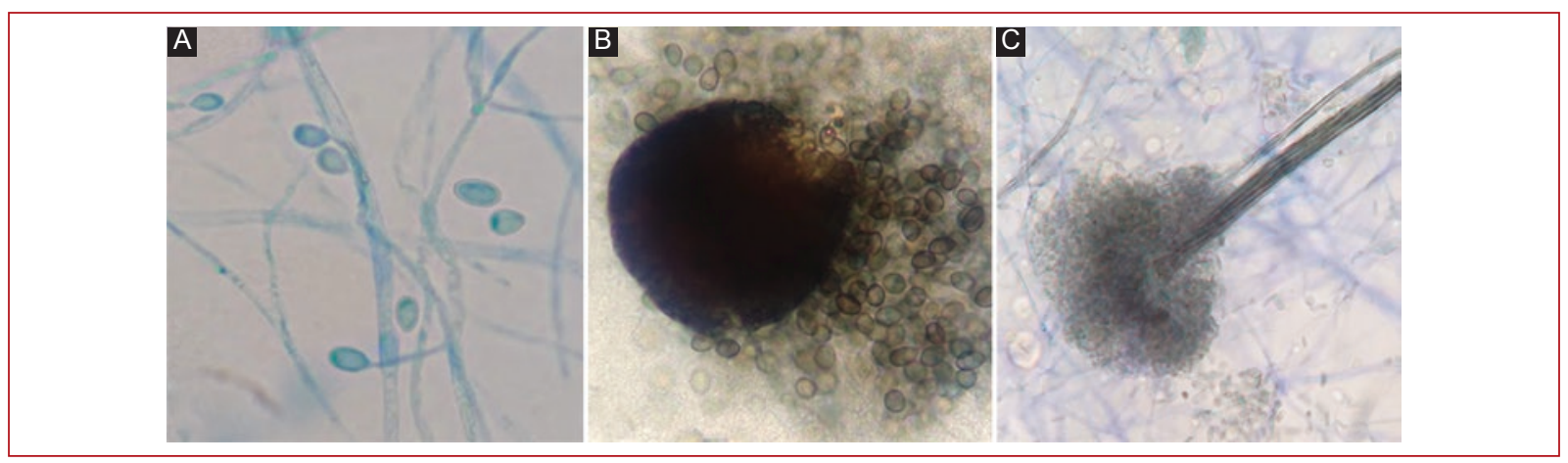

Figure 1. Reproductive structures of the genus Scedosporium. (A) Initial asexual phase: the presence of septate hyaline hyphae with oval conidia. (B) Intermediate sexual phase (in some cases): production of closed ascomas with ascoporas inside. (C) Delayed asexual phase: presence of sinemas with a large number of conidia in the apex.

Table 2. Morphological characteristics of the pathogenic species of the genus Scedosporium

\begin{tabular}{|c|c|c|c|c|}
\hline \multirow[t]{2}{*}{ Species } & \multirow{2}{*}{$\begin{array}{l}\text { Thermotolerance } 45^{\circ} \mathrm{C} \\
\text { present }(+) \text { abscent }(-)\end{array}$} & \multicolumn{3}{|c|}{ Microscopic morphology } \\
\hline & & Conidia & Cleistothecia & Sinema \\
\hline Scedosporium apiospermum & + & Group & + & + \\
\hline Scedosporium boydii & + & Solitary or group & + & + \\
\hline Scedosporium aurantiacum & + & Solitary & - & + \\
\hline Scedosporium dehoogii & - & Solitary & - & - \\
\hline Scedosporium minutisporum & + & Solitary & + & + \\
\hline
\end{tabular}

anaerobic culture conditions, are thermotolerant $\left(45^{\circ} \mathrm{C}\right)$, and also present a tolerance to sodium chloride and magnesium chloride at $5 \%$ concentrations in liquid culture medium ${ }^{2}$. Table 2 shows some characteristics of the different species which confirm this genus.

Macroscopically, in Sabouraud Dextrose Agar culture medium, these fungi develop flat cotton-like or velvety-looking colonies with defined or irregular edges. Initially, the colonies are white, but as they mature, they acquire an olive green to dark gray color. S. aurantiacum is characterized by its ability to produce a diffusible yellow pigment in the conventional culture mediums used in mycology laboratories. Microscopically, Scedosporium spp. produces septated hyaline hyphae with low or clustered short thin conidiophores (Fig. 1A). The conidia are unicellular, with a claviform to globose form and olive or hyaline color.

In a culture medium with low nutritional content such as the potato dextrose agar medium (PDA), they may develop a closed ascoma or cleistothecia immersed in the culture medium in a time lapse of 2-3 weeks of incubation. Ascoma is spherical structures with a yellowish or black color and a diameter of approximately
140-200 $\mu \mathrm{m}$ formed by a single layer of fine flat cells (Fig. 1B). Inside the ascoma, gold unicellular ellipsoid ascospores are developed, which are released once the ascoma is mature ${ }^{2}$. The development of ascoma has been described in $S$. minutisporum, $S$. apiospermum, and S. boydii.

On the other hand, another type of structure called sinema develops usually in the border of the colony when cultures are incubated for a period of time $>3$ weeks. The sinemas are bundles of erect hyphae, yellow or gray, with conidias at the apex (Fig. 1C). These structures have been observed in pathogenic strains of the genus Scedosporium, with the exception of $S$. dehoogii².

\section{Ecology}

The Scedosporium genus includes saprophyte and cosmopolitan fungi, which are found in the soils of regions with temperate and tropical weather. Its isolation is more frequent in soils with high human activity, i.e., agricultural soil, urban gardens, playgrounds, sports gardens, industrial areas, and domestic gardens. This could 
be due to organic pollution and a high amount of nitrogen components in these environments ${ }^{3}$.

In recent years, different research groups have been directed toward establishing whether or not there is a specific ecological niche for each one of the species of this genus. Kalteis et al. conducted a study in Austria and the Netherlands, in which they took samples from soils with high human activity, as well as in natural areas such as the woods, wetlands, and sludge (sea and mud). Interestingly, none of the Scedosporium species were isolated in the samples taken from natural areas. S. apiospermum was the most abundant species in areas with high human activity, present in $58.7 \%-77.7 \%$ of samples, followed by $S$. dehoogii with a proportion of $13.8 \%-28.8 \%$. On the other hand, S. aurantiacum was only found in $4.8-7.7 \%$ of the samples and S. minutisporum was only recovered in $5.8 \%$. In a revision of the clinical cases of scedosporiosis occurred in these countries, a total of 179 reports were found. However, $S$. apiospermum was the main agent isolated in $57 \%$ of the cases, followed by $S$. boydii in $33.5 \%, S$. aurantiacum in $6.1 \%$, and S. minutisporum in $2.2 \%$ of the cases. S. dehoogii was only isolated in two cases of superficial infections ${ }^{3}$.

In France, patients with cystic fibrosis are the population most affected by this fungus ${ }^{4}$. S. boydii was the microorganism most frequently isolated in $62 \%$ of samples of sputum and bronchial aspiration, followed by $S$. apiospermum in $24 \%, S$. aurantiacum in $10 \%$, and $S$. minutisporum in $4 \% 5$.

Due to the high incidence of this fungus among these patients, a study was conducted on the distribution of this fungus in different soils with high human activity. Findings show that $S$. dehoogii was the most abundant species with $43.6 \%$ of isolates, followed by $S$. aurantiacum with $22.3 \%$, S. boydii with $20.3 \%$, and S. apiospermum with $19.5 \%$. S. minutisporum was the least isolated one with $0.3 \%{ }^{6}$. Interestingly, $S$. dehoogii was not recovered from clinical samples despite being the most frequently isolated species in the soils of this country ${ }^{5}$.

In Australia, a similar situation to the one mentioned in France occurs. One in every six patients with cystic fibrosis suffered from a colonization/infection by this fungal group, having $S$. aurantiacum as the most predominant species ${ }^{7}$. Harun et al. researched the prevalence of these fungi in the soils of an urban zone in Sydney. Interestingly, S. aurantiacum was the predominant species with $54.6 \%$, followed by Lomentospora prolificans with $43 \%$, S. boydii with $2.1 \%$, and S. dehoogii with $0.3 \%{ }^{8}$.
In Thailand, after the tsunami in 2004, two infection cases caused by $S$. apiospermum were reported ${ }^{9}$. In 2016, Luplertlop et al. conducted an environmental study in soil and water samples from public parks isolating $S$. apiospermum as the most frequent species with $72.4 \%$, followed by $S$. aurantiacum with $6.1 \%$ and S. dehoogii with $5.1 \%{ }^{10}$.

In Chile, clinical cases related to these fungi are rare. There are only reports of two clinical cases of rhinosinusitis where $S$. boydii and $S$. apiospermum were recovered. However, when a study was conducted on the prevalence of these fungi in soil samples of urban areas in the city of Arica, Valparaiso, and Puerto Varas, only four isolates of $S$. dehoogii were obtained ${ }^{11}$.

In Mexico, the reports of clinical cases are very similar to those published in Chile. Currently, there are only three published cases of mycetoma ${ }^{12}$ and one case of endoftalmitis ${ }^{13}$ caused by S. boydii. In 2017, Elizondo-Zertuche et al. conducted a study to discover the prevalence of these fungi in soils from different country regions. Soils with high human activity were analyzed obtaining a total of 100 isolates, from which $S$. apiospermum was the most predominant species with $69 \%$, followed by S. boydii with $16 \%$, S. aurantiacum with $7 \%$, S. dehoogii with $5 \%$, and S. angustum with $3 \%{ }^{14}$.

\section{Epidemiology}

The epidemiology of these infections is still unknown; nevertheless, in recent years, the number of multicentered studies related to scedosporiosis in the different populations linked to risk factors has increased. Alarmingly, in the past decade, reports of scedosporiosis cases have increased in Europe, America, Asia, and Oceania $^{15}$, as shown in Figure 2.

In 2015, a multicenter study took place in Australia in 15 third-level hospitals to determine the epidemiology of the different invasive infections due to filamentous fungi other than Aspergillus. Of a total of 162 patients, 165 isolates of filamentous fungi were identified with mucoromycetes and Scedosporium spp. being the most common at $45.7 \%$ and $33.3 \%$, respectively ${ }^{16}$.

Patients with cystic fibrosis present frequent colonization in the lungs with Scedosporium spp. with a prevalence between $3.4 \%$ and $17.4 \%$ in Europe ${ }^{17}$, US ${ }^{18}$, and Australia ${ }^{8}$.

The species of Scedosporium are recognized as significant pathogenic agents in solid organ transplants and hematopoietic stem cell transplant. In Spain, Husain et al. conducted a review of cases of organ 


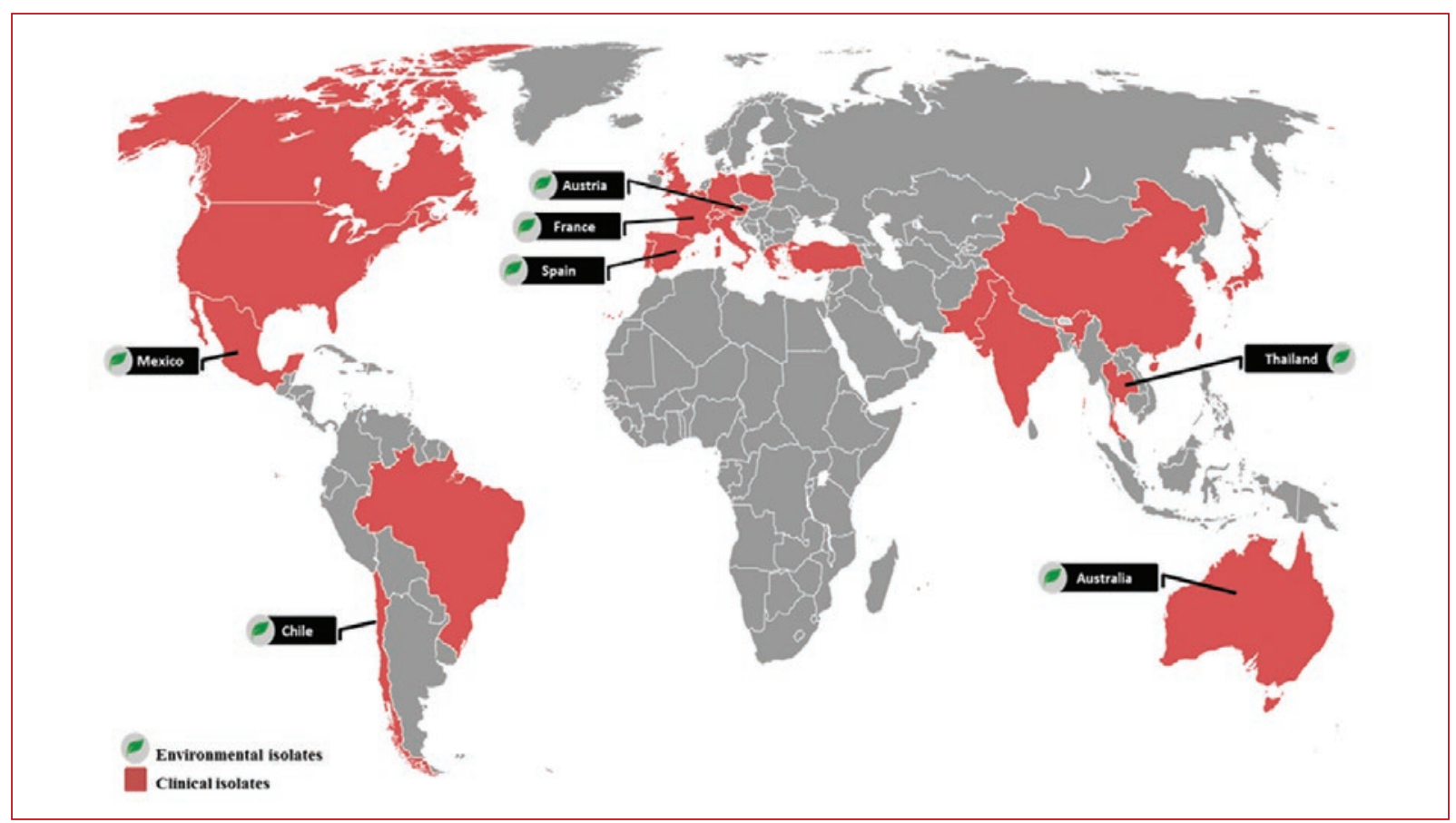

Figure 2. Global distribution of environmental isolations and clinical cases of Scedosporium spp.

receptors with a report of infection by Scedosporium sp. finding a total of 80 cases, with 57 cases of solid organ transplants and 23 cases of hematopoietic stem cell transplant. The organs involved in the infections of the solid organ receptors were the kidney $(35 \%)$, heart (28\%), liver (18\%), lungs (14\%), and small intestine (5\%). Of these patients, $18 \%$ received prophylactic antifungal treatment. S. apiospermum was the pathogenic agent in $83 \%$ of the receptors of solid organs and L. prolificans was the pathogenic agent in $19 \%$ of these cases. In the cases involving hematopoietic stem cell receptors, $64 \%$ received prophylactic antifungal treatment and S. apiospermum was the pathogenic agent in 39\% of these cases ${ }^{19}$.

In the US, a prospective surveillance study for invasive fungal infections was carried out in 23 transplant centers during the period of 2001-2006. There were 169 identified cases of infection by filamentous fungi: 105 by mucorales, 37 by Fusarium sp., and 27 by Scedosporium sp. Of these infections, $73.4 \%$ occurred in hematopoietic stem cell recipients and $26.4 \%$ in solid organ receptors ${ }^{20}$.

In a literature review conducted in Japan, they found 28 cases with scedosporiosis that presented skin manifestations (pustules and nodules), of which only eight patients had a history of trauma. The pathogen in 22 of the 28 cases was $S$. apiospermum ${ }^{21}$.
Outbreaks caused by a pathogenic species of this genus had not been identified although it has been known that these fungi have a predilection for soils with high human activity. In 2015 in Germany, Bernhardt et al. published the first report of a group of infections associated with $S$. boydii in five of six patients who had undergone cardiac surgery in the same surgery unit ${ }^{22}$.

In natural disasters, a situation known as "near-drowning" may occur. This condition is defined as a severe episode of involuntary immersion and aspiration of fluids that justifies hospitalization. It is estimated that this occurrence is 2-20 times more frequent than drowning (according to the WHO, about 372,000 people drown annually around the world). One of the complications of this condition is infection, which can have a fatal outcome. In this regard, the morbidity and mortality associated with infections in victims who have been close to drowning are unknown. Some microorganisms considered as harmless saprophytes have been reported with some frequency as a cause of severe or lethal infection, even in immunocompetent individuals.

Katragkou et al., in a retrospective review of the period from 1950 to 2006, highlighted the importance of scedosporiosis after a near-drowning event. In this study, they found 23 clinical cases of scedosporiosis derived from natural disasters that occurred in 13 countries, such as floods or tsunamis. $S$. apiospermum was the pathogen 
in 21 of the 23 reported cases. S. aurantiacum and $S$. boydii were the pathogens isolated in the two remaining cases. The reported mortality was $70 \%{ }^{23}$.

In Brazil, Oliveira et al. conducted a search of reported clinical cases of scedosporiosis from 1982 to 2013. In this revision, 15 cases of subcutaneous, pulmonary, and in the central nervous system scedosporiosis were found, linked to $S$. apiospermum. The most frequent clinical manifestation was the presence of a fungus ball (33.3\%), mainly in patients previously recovered from tuberculosis. On the other hand, there was a report in the same study of the first case of infection of the central nervous system secondary to a near-drowning ${ }^{24}$.

\section{Clinical presentations}

Transmission of these infections is mainly through trauma, surgeries, and aspiration of contaminated water. The anatomic places where the different species of the Scedosporium genus have been isolated are diverse. To this day, there is no proof that this microorganism has a predilection for a specific anatomic site (Fig. 3).

Localized infections, mainly mycetomas, are among the most frequent cases of reported scedosporiosis. Mycetoma is a chronic suppurative infection of the skin and subcutaneous tissue, which affects the lower limbs in over $80 \%$ of the cases. The condition is characterized by a symptomatic triad consisting of swelling of the affected zone, fistula formation, and purulent discharge containing grains. These infections are produced as a result of the inoculation of the microorganism through skin trauma. The eumycotic mycetomas are caused mainly by Madurella mycetomatis, Trematosphaeria grisea, and S. apiospermum ${ }^{25}$.

The acquisition of an infection by Scedosporium spp. after the introduction of a contaminated foreign body may occur in the eyes, developing keratitis or even endophthalmitis. Although uncommon, there have been reports of otitis caused mainly by $S$. apiospermum ${ }^{26}$.

Scedosporiosis is considered to be a major cause of death in solid organ and hematopoietic stem cell receptors, making it the third most common transplant-related pathogen filamentous fungi after mucorales and Fusarium spp. The most frequent etiologic agent isolated among this population of patients is $S$. apiospermum ${ }^{19}$.

Colonization of the airways, paranasal sinuses, and pulmonary infections has been linked to Scedosporium species. These infections occur, especially, in patients with preexisting pulmonary cavities (bronchiectasis, tuberculosis, sarcoidosis, cystic fibrosis, etc.), developing "fungus balls" in some cases, which are radiologically

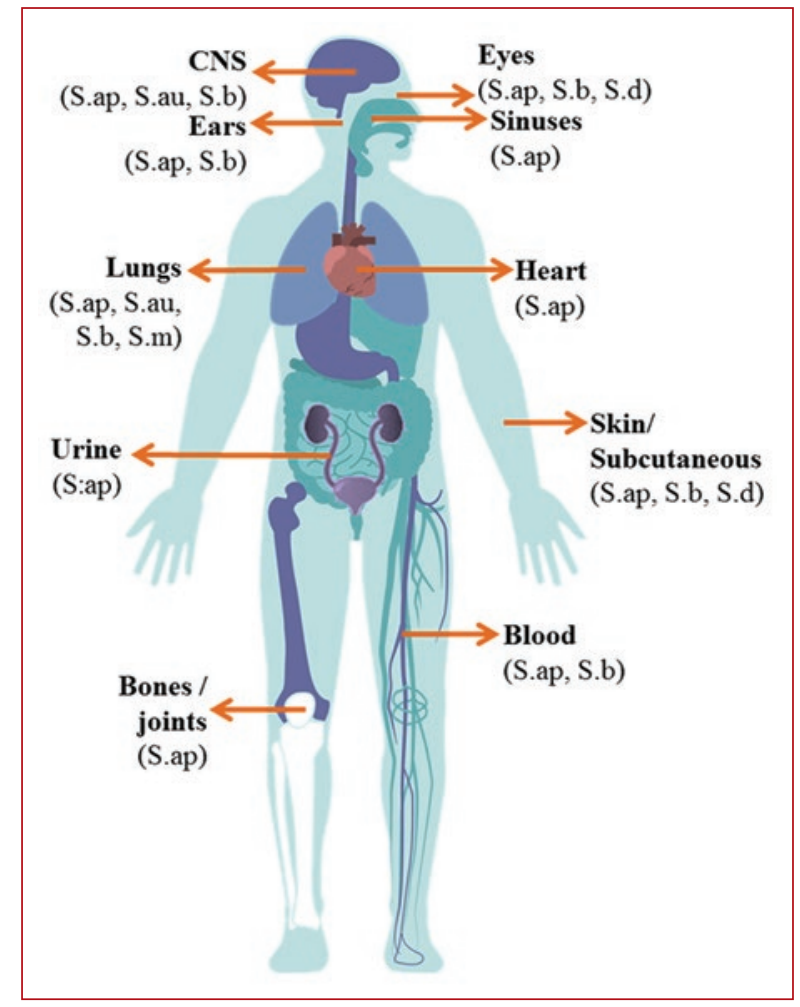

Figure 3. Anatomical sites where the pathogenic species of the genus Scedosporium have been isolated:

Scedosporium apiospermum, Scedosporium boydii, Scedosporium aurantiacum, Scedosporium dehoogii, and Scedosporium minutisporum.

indistinguishable from an aspergilloma ${ }^{27}$. In patients with cystic fibrosis, these fungi are placed as the second filamentous fungi after Aspergillus to colonize the lungs of these individuals, causing allergic bronchopulmonary mycosis as the mildest manifestation, up to a disseminated pulmonary invasive infection ${ }^{28}$.

In individuals with a debilitated immune system such as patients with HIV/AIDS, it is acknowledged that a $\mathrm{CD}^{+}$cell count of $<100 / \mu \mathrm{l}$ is a predisposing factor of an invasive scedosporiosis and that patients with dissemination to the central nervous system usually show a $\mathrm{CD} 4^{+}$cell count of $<50 / \mu \mathrm{l}$. These findings were reported in the exhaustive revision conducted by Tammer et al. in relation to the cases of pseudallescheriosis/ scedosporiosis in patients with HIV during the period of 1990-2010. In this study, there were 14 reports of infection by $P$. boydii or $S$. apiospermum in this HIV-positive population ${ }^{29}$.

In healthy patients who suffered an event of near-drowning, severe clinical conditions are often reported. Aspiration of contaminated water through 
the respiratory tract, with significant amounts of conidia, initially offsets a pulmonary infection. In these situations, clinical symptoms can occur from a few days to several weeks after the incident. Fever is frequently reported, along with productive cough, hemoptysis, wheezing, and rhonchi. Radiographic findings of the thorax can vary, ranging from unilateral focal infiltrates to diffuse bilateral infiltrates and from nodes to bronchopneumonia ${ }^{30}$. Hematogenous dissemination starting in the lungs may manifest on the skin with a maculopapular eruption or as nodular lesions, which may enlarge and become necrotic ${ }^{25}$. An invasion of the central nervous system can manifest as a cerebral abscess (in approximately $77 \%$ of cases), meningitis, encephalitis, ventriculitis, or vascular affectation, which produces sinus thrombosis and stroke ${ }^{23}$.

There are several isolated clinical cases of scedosporiosis in patients with diverse diseases and under varied therapeutic regimes. The case of a patient with a vulgar psoriasis diagnosis has been reported, a patient who, years before, had been diagnosed with erythrodermic psoriasis. This patient was treated with prednisone and cyclosporine, developing nodes and abscesses in both the knees with an absence of fistulae and granules. Mycological laboratory tests were positive. The fungus was identified as $S$. dehoogii using the $\beta$-tubulin gene sequence. The patient rejected undergoing antifungal therapy; however, the lesions disappeared after the removal of cyclosporine and a decrease of the prednisone dose, and there were no relapses during the next 64 months of follow-up. In this paper, the authors conclude that the spontaneous recovery may have come as a result of the immune reconstitution after the immunosuppression was discontinued ${ }^{31}$.

Another interesting case was the report of a patient with a painful swelling and sensitivity in the back of the hand and left wrist, with an evolution of 2 months. Physical examination revealed a fluctuating erythematosus weal, poorly defined, with pustules and scabs. Aspiration of the lesion and biopsy was taken to conduct mycological and histological studies. A fungal colony initially identified as Alternaria spp. was obtained. The sequence analyses of the internal transcribed spacer (ITS) and D1/D2 ribosomal regions were performed, identifying the fungus as $S$. apiospermum. The patient was treated with debridement and fluconazole for 4 weeks, and at the last follow-up appointment 4 months after treatment, the patient had recovered and did not present recurrences ${ }^{32}$.

\section{Diagnosis}

Clinical diagnosis of scedosporiosis continues to be a challenge, since in most cases, signs and symptoms are non-specific, resulting in the suggestion of other fungal etiological agents which occur with more frequency in human pathology.

\section{Direct microscopy and histopathology}

In most cases, direct microscopic and histopathological studies are very useful since they may serve as evidence of a presumptive diagnosis of a fungal infection. However, these studies may not precisely identify the etiological agent of a fungal disease. It is important to remember that a negative result should not rule out the possibility of a mycotic infection.

The direct microscopic examination involves the use of potassium hydroxide at $10 \%$ or the use of fluorescent substances such as Blankophor, Uvitex 2B, or Calcofluor White Stain ${ }^{27}$. In tissue, Scedosporium sp. presents hyaline septate hyphae, which cannot be differentiated from other hyaline fungi such as Aspergillus spp. or Fusarium spp. The hyphae have dichotomic ramifications, which may present intercalated chlamydospores. Rarely, pigmented oval conidia may be observed in tissue $^{25}$. A positive result should always be considered significant, even if it is not possible to obtain a culture from the samples.

In biopsy histopathological examinations, initial staining is hematoxylin and eosin. This stain is used to observe tissue morphology, yet it is of limited usefulness for the visualization of fungal structures, since they may be confused with artifacts, fibrin, or necrotic masses. Thus, there are histochemical techniques which facilitate the visualization of hyphae in tissue, such as periodic acid-Schiff stain, Gridley stain, or the silver methenamine technique (Gomori-Grocott) ${ }^{33}$.

Thornton et al. developed two monoclonal antibodies (HG12 and GA3) to distinguish Scedosporium from other hyaline fungi through immunofluorescence. These antibodies recognize a carbohydrate epitope of an unidentified antigen present in the cell wall of conidia and hyphae, which are specific to $S$. apiospermum, $S$. boydii, and $S$. aurantiacum. While some cross reactions were observed with antigens of Pseudallescheria africana and Lophotrichous fineti, these monoclonal antibodies may be used to differentiate scedosporiosis from an infection by other hyaline fungi ${ }^{34}$. 


\section{Culture}

Scedosporium grows well in conventional culture media for bacteria and fungi (blood-agar, chocolate-agar, Sabouraud dextrose agar, PDA, and yeast extract peptone dextrose). Optimal incubation temperature for the growth of these fungi is between $25^{\circ} \mathrm{C}$ and $35^{\circ} \mathrm{C}$. The growth of these fungi is easily overshadowed by other fast-growing fungi such as Aspergillus; therefore, several semi-selective culture media have been developed for Scedosporium spp.: YPD complemented with cycloheximide, SceSel+, and Scedo-Select III ${ }^{35}$. These media greatly facilitate the growth and recovery of the etiologic agent in clinical samples, especially of respiratory secretions. Although these culture media facilitate the isolation of these fungi, they do not allow their precise identification.

\section{Proteomics}

Mass spectrometry (MALDI-TOF MS, matrix-assisted laser desorption/ionization time-of-flight mass spectrometry) is a technique recently used in the identification of microorganisms through the creation of a spectrum based on protein profiles, which is unique for a species in particular. In the last decade, this technique has been utilized with more frequency in the identification of clinical species. Protein profiles for the identification of the most common pathogenic filamentous fungi are available but not routinely performed in many hospital centers. Even though commercial systems easily identify filamentous fungi frequently isolated from in clinical samples, they are not equipped to properly differentiate closely related fungi groups. Therefore, research has been oriented to create individual databases for specific taxonomic groups of interest. Nevertheless, to date, there is no universal repository for said databases, and they have to be obtained from the authors of their respective publications and evaluated locally.

Recently, MALDI-TOF MS has been tested for the identification of Scedosporium spp. According to the new proposed nomenclature, a clear distinction can be made between two groups: those which cannot be distinguished using this technique (S. apiospermum, S. boydii, S. ellipsoideum, and $S$. angustum) and the species which present a clear distinction among each other (S. aurantiacum and $L$. prolificans) $)^{36}$. The lack of a trustworthy database for the species of this complex has hampered the application of this technique for the identification of Scedosporium spp.

\section{Molecular biology techniques}

A wide range of molecular techniques based on DNA amplification by the polymerase chain reaction (PCR) technique has been used in the identification of the Scedosporium spp. species. Examples of these are the PCR reverse-based line hybridization and restriction fragment length polymorphism ${ }^{37}$. Despite being able to identify the most frequent pathogen agents ( $S$. apiospermum, S. aurantiacum, and L. prolificans), they present drawbacks as they are unable to precisely distinguish between the species of the $S$. apiospermum complex (S. apiospermum, S. boydii, S. angustum, S. ellipsoideum, and $S$. fusoidea).

On the other hand, techniques such as the loop-mediated isothermal amplification ${ }^{38}$, amplified fragment length polymorphism ${ }^{37}$, and the rolling circle amplification $^{39}$ are techniques which have accurately accomplished the identification of the Scedosporium sp. species. The use of these techniques is not yet standardized for routinely diagnostic use.

In 2015, the International Society of Human and Animal Mycology established a DNA barcoding for the identification of fungi of medical importance. A DNA barcoding is a short (500-800 pb) genetically diverse sequence, which is flanked by a known preserved region, allowing for the design of universal oligonucleotides. The sequence of the ITS region of the ribosomal DNA allows for the identification of organisms in a quick, effective, universal, and accessible way, without the need for experts. In the case of closely related groups, an additional gene may be required to discern between the species of the group. For the identification of $S$. aurantiacum and $S$. minutisporum, sequencing of the ITS region is enough; however, for the identification of $S$. apiospermum, $S$. boydii, $S$ ellipsoideum, $S$. angustum, and $S$. dehoogii, an additional sequencing of the $\beta$-tubulin (BT2) is required ${ }^{40}$.

\section{Antifungal susceptibility}

This fungus presents variable behavior against the different antifungals tested in vitro since certain strains present an intrinsic resistance to a wide variety of antifungals. In a bibliographic review on in vitro susceptibility of the species of the Scedosporium genus where 521 isolations of clinical and environmental origins were analyzed, in vitro susceptibility against itraconazole, posaconazole, and voriconazole was highly variable, with minimum inhibitory concentrations (MIC) ranging from 0.016 to $>16 \mathrm{mg} / \mathrm{L}$. The lowest values of 
MIC were frequently observed for voriconazole. P. ellipsoidea, S. minutisporum, S. aurantiacum, S. boydii, and $S$. dehoogii presented less susceptibility for posaconazole, with a MIC range of $0.5->16 \mathrm{mg} / \mathrm{L}^{41}$. Voriconazole was the only triazole showing antifungal activity against $S$. autantiacum, with a MIC range of 0.5-1 $\mu \mathrm{g} / \mathrm{mL}$, while amphotericin B, itraconazole, caspofungin, micafungin, isavuconazole, and anidulafungin did not display antifungal activity against this species. $S$. dehoogii was the most resistant against voriconazole, with a MIC value of $>16 \mu \mathrm{g} / \mathrm{mL}$, while $S$. angustum presented a resistance to isavuconazole, itraconazole, and anidulafungin ${ }^{42}$.

$S$. apiospermum isolates have been classified as susceptible to miconazole, voriconazole, and posaconazole and resistant to fluconazole and flucytosine. A variable susceptibility to ketoconazole, itraconazole, and amphotericin B was observed. Micafungin is the most active echinocandin against this fungus ${ }^{43}$.

\section{Treatment}

Most international guidelines recommend voriconazole as the first-line therapy in a scedosporiosis. In the retrospective revision of Troke et al., a successful therapeutic response was accomplished in $57 \%$ of 107 patients with scedosporiosis. All patients received an initial dose of voriconazole of $6 \mathrm{mg} / \mathrm{kg}$ intravenously, twice a day on day 1 , followed by $4 \mathrm{mg} / \mathrm{kg}$ intravenously twice a day, and later a change to oral therapy of $200 \mathrm{mg}$ twice a day. The total duration of the therapy with voriconazole varied from 1 to 802 days. The best therapeutic responses were observed for cutaneous/ subcutaneous infections $(91 \%)$ or bone infections $(79 \%)$ and the lowest for infections of the central nervous system $(43 \%)^{44}$.

Antifungal combined therapy has emerged as a promising option. Goldman et al. described a case of an immunocompromised patient with a cutaneous infection by $S$. apiospermum, which disseminated despite treatment with voriconazole. Addition of micafungin and the granulocyte macrophage colony-stimulating factor provided a partial recovery, which indicates a possible synergic role for the combined therapy and use of immunotherapeutic agents ${ }^{45}$.

\section{Conclusion}

Emergent fungal infections have increased over the years. Changes in mycologic taxonomy as well as the onset of new species responsible for infections in humans have increased the number of fungi of medical significance.

Today, the role of the species of the Scedosporium genus in the development of invasive infections with a less than favorable prognosis may be underestimated, due to the restrictions of the diagnostic techniques routinely employed.

\section{Conflicts of interest}

The authors have no conflicts of interest to declare.

\section{Financing}

No financial support was provided.

\section{Ethical disclosures}

Protection of human beings and animals. The authors declare that there were no experiments conducted on humans or animals for this study.

Data confidentiality. The authors declare that there are no patient data in this article.

Right to privacy and informed consent. The authors declare that there are no patient data within this article.

\section{References}

1. Steinbach WJ, Perfect JR. Scedosporium species infections and treatments. J Chemother. 2003;15 Suppl 2:16-27.

2. Cortez KJ, Roilides E, Quiroz-Telles F, et al. Infections caused by Scedosporium Spp. Clin Microbiol Rev. 2008;21:157-97.

3. Kaltseis J, Rainer J, De Hoog GS. Ecology of Pseudallescheria and Scedosporium species in human-dominated and natural environments and their distribution in clinical samples. Med Mycol. 2009;47:398-405.

4. Parize P, Billaud S, Bienvenu AL, et al. Impact of Scedosporium apiospermum complex seroprevalence in patients with cystic fibrosis. J Cyst Fibros. 2014;13:667-73.

5. Zouhair R, Rougeron A, Razafimandimby B, et al. Distribution of the different species of the Pseudallescheria boydii/Scedosporium apiospermum complex in French patients with cystic fibrosis. Med Mycol. 2013;51: 603-13.

6. Rougeron A, Schuliar G, Leto J, et al. Human-impacted areas of France are environmental reservoirs of the Pseudallescheria boydii/Scedosporium apiospermum species complex. Environ Microbiol. 2015;17:1039-48.

7. Blyth CC, Middleton PG, Harun A, et al. Clinical associations and prevalence of Scedosporium Spp. In Australian cystic fibrosis patients: identification of novel risk factors? Med Mycol. 2010;48 Suppl 1:S37-44.

8. Harun A, Gilgado F, Chen SC, Meyer W. Abundance of Pseudallescheria/Scedosporium species in the Australian urban environment suggests a possible source for scedosporiosis including the colonization of airways in cystic fibrosis. Med Mycol. 2010;48 Suppl 1:S70-6.

9. Garzoni C, Emonet S, Legout L, et al. Atypical infections in tsunami survivors. Emerg Infect Dis. 2005;11:1591-3.

10. Luplertlop N, Pumeesat $P$, Muangkaew W, Wongsuk T, Alastruey-Izquierdo A. Environmental screening for the Scedosporium apiospermum species complex in public parks in Bangkok, Thailand. PLoS One. 2016;11: e0159869.

11. Alvarez E, Sanhueza C. New record of Scedosporium dehoogii from Chile: phylogeny and susceptibility profiles to classic and novel putative antifungal agents. Rev Iberoam Micol. 2016;33:224-9.

12. Bonifaz A, Tirado-Sánchez A, Calderón L, et al. Mycetoma: experience of 482 cases in a single center in Mexico. PLoS Negl Trop Dis. 2014; 8:e3102. 
13. Vanzzini-Zago V, Corredor-Casas S, Rodríguez-Reyes A, et al. Endoftalmitis de probable origen endógeno causada por Scedosporium boydii. Informe de un caso. Rev Iberoam Micol. 2016;33:122-5.

14. Elizondo-Zertuche M, Treviño-Rangel RJ, Robledo-Leal E, et al. Molecular identification and in vitro antifungal susceptibility of Scedosporium complex isolates from high-human-activity sites in Mexico. Mycologia. 2017; $109: 874-81$.

15. Luplertlop N. Pseudallescheria/Scedosporium complex species: from saprobic to pathogenic fungus. J Mycol Med. 2018;28:249-56.

16. Slavin M, van Hal S, Sorrell TC, et al. Invasive infections due to filamentous fungi other than Aspergillus: epidemiology and determinants of mortality. Clin Microbiol Infect. 2015;21:490.e1-10.

17. Sedlacek L, Graf B, Schwarz C, et al. Prevalence of Scedosporium species and Lomentospora prolificans in patients with cystic fibrosis in a multicenter trial by use of a selective medium. J Cyst Fibros. 2015;14:237-41.

18. Hong G, Miller HB, Allgood S, et al. Use of selective fungal culture media increases rates of detection of fungi in the respiratory tract of cystic fibrosis patients. J Clin Microbiol. 2017;55:1122-30.

19. Husain S, Muñoz P, Forrest G, et al. Infections due to Scedosporium apiospermum and Scedosporium prolificans in transplant recipients: clinical characteristics and impact of antifungal agent therapy on outcome. Clin Infect Dis. 2005;40:89-99.

20. Park BJ, Pappas PG, Wannemuehler KA, et al. Invasive non Aspergillus mold infections in transplant recipients, United States, 2001-2006. Emerg Infect Dis. 2011;17:1855-64.

21. Ishii S, Hiruma M, Hayakawa Y, et al. Cutaneous Pseudallescheria boydii/Scedosporium apiospermum complex (Molecular type: Scedosporium apiospermum [Clade 4]) infection: a case report and literature review of cases from Japan. Med Mycol J. 2015;56:E25-30.

22. Bernhardt A, Seibold M, Rickerts V, Tintelnot K. Cluster analysis of Scedosporium boydii infections in a single hospital. Int J Med Microbiol. 2015;305:724-8.

23. Katragkou A, Dotis J, Kotsiou M, Tamiolaki M, Roilides E. Scedosporium apiospermum infection after near-drowning. Mycoses. 2007;50:412-21.

24. Oliveira Fde M, Unis G, Hochhegger B, Severo LC. Scedosporium apiospermum eumycetoma successfully treated with oral voriconazole: report of a case and review of the Brazilian reports on scedosporiosis. Rev Inst Med Trop Sao Paulo. 2013;55:121-3.

25. Cortez KJ, Roilides E, Quiroz-Telles F, et al. Infections caused by Scedosporium Spp. Clin Microbiol Rev. 2008;21:157-97.

26. Bhally HS, Shields C, Lin SY, Merz WG. Otitis caused by Scedosporium apiospermum in an immunocompetent child. Int J Pediatr Otorhinolaryngol. 2004;68:975-8

27. Giraud S, Bouchara JP. Scedosporium apiospermum complex: diagnosis and species identification. Curr Fungal Infect Rep. 2014;8:211-9.

28. Cimon B, Carrère J, Vinatier JF, et al. Clinical significance of Scedosporium apiospermum in patients with cystic fibrosis. Eur $\mathrm{J}$ Clin Microbio Infect Dis. 2000;19:53-6.

29. Tammer I, Tintelnot K, Braun-Dullaeus RC, et al.Infections due to Pseudallescheria/Scedosporium species in patients with advanced HIV disease a diagnostic and therapeutic challenge. Int J Infect Dis. 2011;15:e422-9.
30. Nakamura Y, Suzuki N, Nakajima Y, et al. Scedosporium aurantiacum brain abscess after near-drowning in a survivor of a tsunami in Japan. Respir Investig. 2013;51:207-11.

31. Li FG, Yang YP, Li W, et al. Spontaneous remission of subcutaneous scedosporiosis caused by Scedosporium dehoogii in a psoriatic patient. Mycopathologia. 2017;182:561-7.

32. Kim CM, Lim SC, Kim J, et al. Tenosynovitis caused by Scedosporium apiospermum infection misdiagnosed as an Alternaria species: a case report. BMC Infect Dis. 2017;17:72.

33. Guarner J, Brandt ME. Histopathologic diagnosis of fungal infections in the $21^{\text {st }}$ century. Clin Microbiol Rev. 2011;24:247-80.

34. Thornton CR. Tracking the emerging human pathogen Pseudallescheria boydii by using highly specific monoclonal antibodies. Clin Vaccine Immunol. 2009;16:756-64.

35. Pham T, Giraud S, Schuliar G, Rougeron A, Bouchara JP. Scedo-select III: a new semi-selective culture medium for detection of the Scedosporium apiospermum species complex. Med Mycol. 2015;53:512-9.

36. Bernhard M, Zautner AE, Steinmann J, et al. Towards proteomic species barcoding of fungi an example using Scedosporium/Pseudallescheria complex isolates. Fungal Biol. 2016;120:162-5.

37. Lackner M, Hagen F, Meis JF, et al. Susceptibility and diversity in the therapy-refractory genus Scedosporium. Antimicrob Agents Chemother. 2014;58:5877-85

38. Lu Q, van den Ende AH, Bakkers JM, et al. Identification of Pseudallescheria and Scedosporium species by three molecular methods. J Clin Microbiol. 2011;49:960-7.

39. Meyer W, Guarro J. Current status in diagnosis of Scedosporium infections: what is the impact of new molecular methods? Curr Fungal Infect Rep. 2014;8:220-6.

40. Ramirez-Garcia A, Pellon A, Rementeria A, et al. Scedosporium and Lomentospora: an updated overview of underrated opportunists. Med Mycol. 2018;56:102-25

41. Araujo R, Oliveira M, Amorim A, Sampaio-Maia B. Unpredictable susceptibility of emerging clinical moulds to tri-azoles: review of the literature and upcoming challenges for mould identification. Eur J Clin Microbiol Infect Dis. 2015;34:1289-301.

42. Lackner M, de Hoog GS, Verweij PE, et al. Species-specific antifungal susceptibility patterns of Scedosporium and Pseudallescheria species. Antimicrob Agents Chemother. 2012:56:2635-42.

43. Lackner M, Fernández-Silva F, Guarro J, Lass-Flörl C. Assessing micafungin/triazole combinations for the treatment of invasive scedosporiosis due to Scedosporium apiospermum and Scedosporium boydii. J Antimicrob Chemother. 2014;69:3027-32.

44. Troke P, Aguirrebengoa K, Arteaga $C$, et al. Treatment of scedosporiosis with voriconazole: clinical experience with 107 patients. Antimicrob Agents Chemother. 2008;52:1743-50.

45. Goldman C, Akiyama MJ, Torres J, Louie E, Meehan SA. Scedosporium apiospermum infections and the role of combination antifungal therapy and GM-CSF: a case report and review of the literature. Med Mycol Case Rep. 2016;11:40-3. 